\title{
Atuação do enfermeiro em distintos modelos de Atenção Primária à Saúde no Brasil
}

\author{
Nurses' performance in distinct models of Primary Health Care in \\ Brazil \\ Beatriz Rosana Gonçalves de Oliveira Toso', Larissa Fungueto', Maristela Salete Maraschin', Nelsi \\ Salete Tonini
}

DOI: $10.1590 / 0103-1104202113008$

RESUMO O estudo teve como objetivo comparar a atuação do enfermeiro em distintos modelos de Atenção Primária à Saúde - saúde da família e atenção tradicional. Estudo observacional, analítico, de corte transversal, em município de médio porte no Oeste do Paraná, realizado no ano de 2017, com 24 enfermeiros de unidades de atenção primária, que responderam a questões oriundas do processo de trabalho constante da Política Nacional de Atenção Básica. Análise dos dados por estatística descritiva. Evidenciou-se que o conjunto das atividades atribuídas ao enfermeiro da atenção primária é amplo, sendo esse profissional responsável por ações gerenciais e assistenciais. Houve semelhança do processo de trabalho nos distintos modelos. Percebeu-se ausência de ações a grupos prioritários e de promoção de saúde. Conclui-se que o enfermeiro enfrenta dificuldades em atuar como profissional integrante das equipes e em otimizar seu tempo entre tarefas organizativas e de cuidado aos indivíduos e populações.

PALAVRAS-CHAVE Enfermagem em saúde pública. Enfermagem. Atenção Primária à Saúde. Estratégia Saúde da Família. Trabalho.

ABSTRACT The study aimed to compare the performance of nurses in different models of Primary Health Care - family health and traditional care. Observational, analytical, cross-sectional study in a medium-sized municipality in western Paraná, in 2017, carried out with 24 nurses from primary care units, who answered questions from the work process contained in the National Primary Care Policy. Data analysis by descriptive statistics. It was evidenced that the set of activities attributed to primary care nurses is broad, and that this professional is responsible for management and care actions. There was similarity of the work process in the different models. The lack of actions to priority and health promotion groups was perceived. In conclusion, the nurse face difficulties in acting as a professional member of the teams and in optimizing their time among organizational and care tasks for individuals and populations.

KEYWORD Public health nursing. Nursing. Primary Health Care. Family Health Strategy. Work.

1 Universidade Estadual do Oeste do Paraná

(Unioeste) - Cascavel (PR), Brasil.

Ib.toso@gmail.com 


\section{Introdução}

A Atenção Primária à Saúde (APS) é reconhecida internacionalmente como importante estratégia para a reorganização e para a ampliação da efetividade dos sistemas de saúde. Representa o primeiro nível de acesso dos usuários ao sistema de saúde diante de necessidades e constitui-se como elemento essencial em um processo continuado de cuidado, desenvolvendo ações e serviços de prevenção, promoção, proteção e reabilitação à saúde, de forma a atender aos problemas de saúde dos indivíduos, famílias e comunidades $\mathbf{1}$.

A Atenção Básica à Saúde (ABS), termo equivalente a APS, é desenvolvida no Brasil seguindo as normas e diretrizes estabelecidas pela Política Nacional da Atenção Básica (PNAB), cujas premissas foram dispostas no Pacto pela Saúde, em 2006, expressas na PNAB de 2011 e mantidas na Portaria ${ }^{\circ} 2.436$, de 21 de setembro de $2017^{2}$.

O cuidado nessa esfera de atenção é oferecido em unidades de saúde em que coexistem dois modelos de atenção, aqui entendidos como equivalentes "a modelo assistencial, referindo-se ao cuidado, à assistência, à intervenção, às ações ou às práticas de saúde"3(547). Ainda, "são uma espécie de lógica que orienta a ação e organiza os meios de trabalho utilizados nas práticas de saúde" ${ }^{3(554)}$, coexistindo no Brasil na Estratégia Saúde da Família (ESF) e nas Unidades Básicas de Saúde (UBS) tradicionais (modelo sanitarista e modelo médico hegemônico, respectivamente). Assim, não se trata de tipologia de serviços. Ao referir-se a Unidades de Saúde da Família (USF) e UBS como modelos distintos, assume-se a sua configuração como modelos de atenção à saúde no Brasil. A implantação da ESF no Brasil apresenta-se com uma cobertura de aproximadamente $60 \%$ do território nacional, e sua existência como modelo orientador do cuidado nesse âmbito de atenção parece ter deixado de ser a meta na nova PNAB, a qual reafirma a coexistência de ambos os modelos ${ }^{2}$. As mudanças introduzidas pela PNAB, em 2017,
Promovem a relativização da cobertura universal, a segmentação do acesso, a recomposição das equipes, a reorganização do processo de trabalho e a fragilização da coordenação nacional da política4(11).

Destarte essa ambiguidade nos modelos de atenção, a prática do enfermeiro na APS, no Brasil, constitui-se como um de seus pilares de funcionamento, sendo sua atuação considerada instrumento de mudanças nas práticas de atenção à saúde, atuando na integralidade do cuidado, na intervenção frente aos fatores de risco, na prevenção de doenças e na promoção da saúde e da qualidade de vida ${ }^{5}$. A profissão enfermagemé socialmente relevante, historicamente determinada e integra o processo de trabalho em saúde, articulado com os demais membros da equipe ${ }^{6}$.

Diante de um cenário de incertezas, tanto do ponto de vista da legislação, do financiamento do SUS, da manutenção de modelos opostos de cuidado quanto do processo de trabalho, esta pesquisa traz questionamentos como: quais são as atividades desenvolvidas pelo enfermeiro nas unidades de APS? Esse profissional atua em consonância com o processo de trabalho disposto na PNAB como atribuição do enfermeiro? Há diferenças no trabalho do enfermeiro decorrentes do modelo de atenção? Para responder a essas questões, o estudo pretendeu abranger as atividades realizadas pelo enfermeiro nos dois modelos de atenção existentes no País, para as unidades de APS.

\section{Objetivo}

Comparar a atuação do enfermeiro em distintos modelos de APS - saúde da família e atenção tradicional

\section{Material e métodos}

Estudo observacional analítico de corte transversal, cujo contexto de desenvolvimento foi 
a área urbana de município de médio porte no Oeste do Paraná, nas 16 UBS e 12 USF, de um total de 28 unidades, cuja coleta de dados ocorreu no segundo semestre de 2017, tanto para o recrutamento quanto para a coleta de dados.

O convite para a participação foi enviado a todos os enfermeiros lotados nas unidades eleitas, sendo aceito por 24 enfermeiros, 12 de UBS e 12 de USF, havendo uma perda por recusa em quatro unidades. Não houve perda por outros motivos. A escolha dos participantes foi intencional, incluindo o total de enfermeiros atuantes nas unidades mencionadas, cujo único critério de inclusão era ser enfermeiro atuante na atenção primária da região urbana do município. Foram excluídos os enfermeiros que atuavam na atenção primária nas unidades rurais e em serviços administrativos da secretaria municipal de saúde.

A coleta de dados foi realizada a partir de roteiro estruturado, elaborado pelas pesquisadoras a partir das atribuições do profissional enfermeiro dispostas na $\mathrm{PNAB}^{2}$. O roteiro foi construído com 67 questões e enviado aos participantes junto ao termo de consentimento, via e-mail, autopreenchido e devolvido. As opções de respostas do formulário se constituíram por três alternativas: sim, não e em parte, referentes à realização das atribuições de cada pergunta, havendo um espaço após cada opção de resposta para comentários, que deixava a critério do participante preencher ou não.

O instrumento foi criado segundo as atribuições apresentadas pela $\mathrm{PNAB}^{2}$ e foi dividido em três partes, sendo: a primeira, as atribuições do enfermeiro comuns aos membros da equipe de saúde; a segunda referente às atribuições específicas do processo de trabalho do enfermeiro; e a terceira sobre os cuidados específicos realizados pelos enfermeiros.

A análise dos dados foi feita por meio de estatística descritiva, com apresentação das frequências relativas e percentuais, com processamento no programa Excel $^{\circledR}$ 2016. Para avaliar a presença de ações do processo de trabalho, utilizou-se a métrica quartílica: (a) executa as ações em acordo com o processo de trabalho - médias de 71 a 100; (b) executa as ações parcialmente em acordo com o processo de trabalho - médias de 50 a 70; (c) não executa as ações em acordo com o processo de trabalho - médias menores que 49.

A pesquisa foi submetida ao Comitê de Ética em Pesquisa com Seres Humanos com número de CAAE: 65448917.2.0000.0107 e aprovada sob parecer $n^{0} 1.999 .973$.

\section{Resultados}

Na tabela 1, descreve-se a participação do enfermeiro nas atribuições comuns a todos os profissionais da APS.

Tabela 1. Atribuições comuns aos membros da equipe de saúde na Atenção Primária à Saúde com participação do enfermeiro, em consonância com o processo de trabalho. Cascavel, Paraná, Brasil. 2017

\begin{tabular}{|c|c|c|c|c|c|c|c|c|c|c|c|c|}
\hline \multirow[b]{3}{*}{ ATIVIDADES } & \multicolumn{6}{|c|}{ Unidade Básica } & \multicolumn{6}{|c|}{ Saúde da Família } \\
\hline & \multicolumn{2}{|c|}{ SIM } & \multicolumn{2}{|c|}{ NÃO } & \multicolumn{2}{|c|}{ Parcial } & \multicolumn{2}{|c|}{ SIM } & \multicolumn{2}{|c|}{ NÃO } & \multicolumn{2}{|c|}{ Parcial } \\
\hline & $\mathbf{N}$ & $\%$ & $\mathbf{N}$ & $\%$ & $\mathbf{N}$ & $\%$ & $\mathbf{N}$ & $\%$ & $\mathbf{N}$ & $\%$ & $\mathbf{N}$ & $\%$ \\
\hline Participar da territorialização & 11 & $92 a$ & 0 & 0 & 1 & 8 & 8 & $67 b$ & 0 & 0 & 4 & 33 \\
\hline $\begin{array}{l}\text { Cuidado integral da saúde da população } \\
\text { adscrita }\end{array}$ & 10 & $83 a$ & 0 & 0 & 2 & 17 & 10 & $83 a$ & 0 & 0 & 2 & 17 \\
\hline Ações de atenção à saúde & 11 & $92 a$ & 0 & 0 & 1 & 8 & 7 & $58 b$ & 0 & 0 & 5 & 42 \\
\hline $\begin{array}{l}\text { Ações de promoção, prevenção, proteção e } \\
\text { recuperação }\end{array}$ & 9 & $75 a$ & 0 & 0 & 3 & 25 & 9 & $75 a$ & 0 & 0 & 2 & 17 \\
\hline
\end{tabular}


Tabela 1. (cont.)

\begin{tabular}{|c|c|c|c|c|c|c|c|c|c|c|c|c|}
\hline \multirow[b]{3}{*}{ ATIVIDADES } & \multicolumn{6}{|c|}{ Unidade Básica } & \multicolumn{6}{|c|}{ Saúde da Família } \\
\hline & \multicolumn{2}{|c|}{ SIM } & \multicolumn{2}{|c|}{ NÃO } & \multicolumn{2}{|c|}{ Parcial } & \multicolumn{2}{|c|}{ SIM } & \multicolumn{2}{|c|}{ NÃO } & \multicolumn{2}{|c|}{ Parcial } \\
\hline & $\mathbf{N}$ & $\%$ & $\mathbf{N}$ & $\%$ & $\mathbf{N}$ & $\%$ & $\mathbf{N}$ & $\%$ & $\mathbf{N}$ & $\%$ & $\mathbf{N}$ & $\%$ \\
\hline Atendimento demanda espontânea & 12 & $100 a$ & 0 & 0 & 0 & 0 & 11 & $92 a$ & 0 & 0 & 1 & 8 \\
\hline Ações programáticas & 10 & $82 a$ & 0 & 0 & 2 & 17 & 11 & $92 a$ & 0 & 0 & 1 & 8 \\
\hline Participa do acolhimento & 8 & $67 b$ & 1 & 8 & 3 & 25 & 12 & $100 a$ & 0 & 0 & 0 & 0 \\
\hline $\begin{array}{l}\text { Identifica necessidade de intervenções de } \\
\text { cuidado }\end{array}$ & 7 & $58 b$ & 0 & 0 & 5 & 42 & 10 & $83 a$ & 0 & 0 & 2 & 17 \\
\hline Realiza busca ativa e notificação & 12 & $100 a$ & 0 & 0 & 0 & 0 & 12 & $100 a$ & 0 & 0 & 0 & 0 \\
\hline Realiza reuniões de equipe & 5 & $42 c$ & 2 & 17 & 5 & 42 & 10 & $83 a$ & 0 & 0 & 2 & 17 \\
\hline Faz registro das atividades & 11 & $92 a$ & 0 & 0 & 1 & 8 & 10 & $83 a$ & 0 & 0 & 2 & 17 \\
\hline Realiza trabalho interdisciplinar & 9 & $75 a$ & 1 & 8 & 2 & 17 & 10 & $83 a$ & 0 & 0 & 2 & 17 \\
\hline Participa de educação permanente & 7 & $58 b$ & 3 & 25 & 2 & 17 & 10 & $83 a$ & 0 & 0 & 2 & 17 \\
\hline
\end{tabular}

Fonte: Elaboração própria.

(a) executa as ações em acordo com o processo de trabalho - médias de 71 a 100; (b) executa as ações parcialmente em acordo com o processo de trabalho - médias de 50 a 70; (c) não executa as ações em acordo com o processo de trabalho - médias menores que 49.

Sobressaíram no processo de trabalho do enfermeiro na UBS ações de territorialização e de atenção à saúde. Na ESF, houve predomínio de acolhimento, identificação de necessidade de intervenções de cuidado, reuniões de equipe e participação em ações de educação permanente em saúde. Foram similares para ambos os modelos: o cuidado da saúde da população adscrita, as ações de promoção, prevenção, proteção e recuperação da saúde, busca ativa e notificação de faltosos, atendimento à demanda espontânea, ações programáticas, trabalho interdisciplinar, além do registro das atividades. A ação que aparece de forma díspar entre as unidades é o cadastro das famílias, pouco desenvolvida em ambas, mas com predominância na ESF.

$\mathrm{Na}$ tabela 2, apresentam-se os dados referentes às atribuições do processo de trabalho específico do enfermeiro na APS.

Tabela 2. Atribuições específicas do processo de trabalho do enfermeiro na Atenção Primária à Saúde. Cascavel, Paraná, Brasil. 2017

\begin{tabular}{|c|c|c|c|c|c|c|c|c|c|c|c|c|}
\hline \multirow[b]{3}{*}{ ATIVIDADES } & \multicolumn{6}{|c|}{ Unidade Básica } & \multicolumn{6}{|c|}{ Saúde da Família } \\
\hline & \multicolumn{2}{|c|}{ SIM } & \multicolumn{2}{|c|}{ NÃO } & \multicolumn{2}{|c|}{ Parcial } & \multicolumn{2}{|c|}{ SIM } & \multicolumn{2}{|c|}{ NÃO } & \multicolumn{2}{|c|}{ Parcial } \\
\hline & $\mathbf{N}$ & $\%$ & $\mathbf{N}$ & $\%$ & $\mathbf{N}$ & $\%$ & $\mathbf{N}$ & $\%$ & $\mathbf{N}$ & $\%$ & $\mathbf{N}$ & $\%$ \\
\hline $\begin{array}{l}\text { Realiza atenção à saúde aos indivíduos e } \\
\text { família no domicílio e em espaços comuni- } \\
\text { tários }\end{array}$ & 10 & $83 a$ & 1 & 8 & 1 & 8 & 10 & $83 a$ & 0 & 0 & 2 & 17 \\
\hline $\begin{array}{l}\text { Realiza Consulta de Enfermagem, procedi- } \\
\text { mentos e atividades em grupo }\end{array}$ & 12 & $100 a$ & 0 & 0 & 0 & 0 & 10 & $83 a$ & 0 & 0 & 2 & 17 \\
\hline Solicita exames complementares & 12 & $100 a$ & 0 & 0 & 0 & 0 & 11 & $92 \mathrm{a}$ & 1 & 8 & 0 & 0 \\
\hline Prescreve medicações & 1 & $8 c$ & 11 & 92 & 0 & 0 & 2 & $17 c$ & 9 & 75 & 1 & 8 \\
\hline Encaminha usuários a outros serviços & 11 & $92 \mathrm{a}$ & 0 & 0 & 1 & 8 & 12 & $100 a$ & 0 & 0 & 0 & 0 \\
\hline
\end{tabular}


Tabela 2. (cont.)

\begin{tabular}{|c|c|c|c|c|c|c|c|c|c|c|c|c|}
\hline \multirow[b]{3}{*}{ ATIVIDADES } & \multicolumn{6}{|c|}{ Unidade Básica } & \multicolumn{6}{|c|}{ Saúde da Família } \\
\hline & \multicolumn{2}{|c|}{ SIM } & \multicolumn{2}{|c|}{ NÃO } & \multicolumn{2}{|c|}{ Parcial } & \multicolumn{2}{|c|}{ SIM } & \multicolumn{2}{|c|}{ NÃO } & \multicolumn{2}{|c|}{ Parcial } \\
\hline & $\mathbf{N}$ & $\%$ & $\mathbf{N}$ & $\%$ & $\mathbf{N}$ & $\%$ & $\mathbf{N}$ & $\%$ & $\mathbf{N}$ & $\%$ & $\mathbf{N}$ & $\%$ \\
\hline $\begin{array}{l}\text { Realiza atividades programadas e à demanda } \\
\text { espontânea }\end{array}$ & 11 & $92 a$ & 0 & 0 & 1 & 8 & 11 & $92 a$ & 0 & 0 & 1 & 8 \\
\hline $\begin{array}{l}\text { Gerencia e avalia ações dos Agentes Comu- } \\
\text { nitários }\end{array}$ & 8 & $67 b$ & 2 & 17 & 2 & 17 & 9 & $75 a$ & 0 & 0 & 3 & 25 \\
\hline $\begin{array}{l}\text { Organiza Educação Permanente com a } \\
\text { equipe }\end{array}$ & 9 & $75 a$ & 2 & 17 & 1 & 8 & 12 & $100 a$ & 0 & 0 & 0 & 0 \\
\hline Participa do gerenciamento de insumos & 9 & $75 a$ & 0 & 0 & 3 & 25 & 10 & $83 a$ & 0 & 0 & 2 & 17 \\
\hline $\begin{array}{l}\text { Possui formação para atenção básica e parti- } \\
\text { cipa de especializações }\end{array}$ & 9 & $75 a$ & 1 & 8 & 2 & 17 & 10 & $83 a$ & 1 & 8 & 1 & 8 \\
\hline $\begin{array}{l}\text { Utiliza de dispositivos de educação para } \\
\text { qualificação do cuidado }\end{array}$ & 3 & $25 c$ & 3 & 25 & 6 & 50 & 5 & $42 c$ & 3 & 25 & 4 & 33 \\
\hline $\begin{array}{l}\text { Trabalha e cria vínculo com o território de- } \\
\text { finido }\end{array}$ & 7 & $58 b$ & 1 & 8 & 4 & 33 & 11 & $92 a$ & 0 & 0 & 1 & 8 \\
\hline Realiza e utiliza diagnóstico situacional & 2 & $17 \mathrm{c}$ & 2 & 17 & 8 & 67 & 2 & $17 c$ & 3 & 25 & 7 & 58 \\
\hline $\begin{array}{l}\text { Organiza o agendamento da população com } \\
\text { a equipe }\end{array}$ & 7 & $58 b$ & 3 & 25 & 2 & 17 & 5 & $42 c$ & 0 & 0 & 7 & 58 \\
\hline $\begin{array}{l}\text { Utiliza ferramentas na gestão do cuidado em } \\
\text { casos complexos }\end{array}$ & 4 & $33 c$ & 3 & 25 & 5 & 42 & 4 & $33 c$ & 1 & 8 & 6 & 50 \\
\hline $\begin{array}{l}\text { Coordena, registra e monitora encaminha- } \\
\text { mentos dos usuários }\end{array}$ & 3 & $25 c$ & 2 & 17 & 7 & 58 & 4 & $33 c$ & 1 & 8 & 6 & 50 \\
\hline $\begin{array}{l}\text { Utiliza estratégias de abordagem familiar e } \\
\text { social }\end{array}$ & 3 & $25 c$ & 3 & 25 & 6 & 50 & 3 & $25 c$ & 0 & 0 & 8 & 67 \\
\hline $\begin{array}{l}\text { Oferece atendimento à população por } 40 \mathrm{~h} \\
\text { semanais }\end{array}$ & 8 & $67 b$ & 2 & 17 & 2 & 17 & 9 & $75 a$ & 1 & 8 & 2 & 17 \\
\hline $\begin{array}{l}\text { Realiza reuniões para monitoramento e } \\
\text { avaliação dos resultados }\end{array}$ & 3 & $25 c$ & 7 & 58 & 2 & 17 & 10 & $83 a$ & 0 & 0 & 2 & 17 \\
\hline $\begin{array}{l}\text { Faz registro no sistema de informação da } \\
\text { atenção básica }\end{array}$ & 4 & $33 c$ & 1 & 8 & 7 & 58 & 8 & $67 b$ & 0 & 0 & 4 & 33 \\
\hline
\end{tabular}

Fonte: Elaboração própria.

(a) executa as ações em acordo com o processo de trabalho - médias de 71 a 100; (b) executa as ações parcialmente em acordo com o processo de trabalho - médias de 50 a 70; (c) não executa as ações em acordo com o processo de trabalho - médias menores que 49.

Na métrica A, sobressaiu a ESF, em atividades típicas desse modelo de cuidado, como o gerenciamento dos Agentes Comunitários de Saúde (ACS), vínculo com território definido, atendimento durante 40 horas semanais, reuniões de monitoramento e avaliação de resultados e registro no Sistema de Informação da Atenção Básica (Siab). De forma similar para ambos os modelos de atenção, destacam-se as ações de saúde aos indivíduos e à família no domicílio, atividades programadas e atenção a demanda espontânea, consulta de enfermagem, solicitação de exames, encaminhamento a outros serviços, educação permanente em saúde, gerenciamento de insumos e formação para a Atenção Básica (AB).

Entretanto, na métrica $\mathrm{C}$, portanto, não convergente com o processo de trabalho, e de forma similar para ambos os modelos de atenção, encontraram-se diagnóstico 
situacional, ferramentas de gestão de caso e uso de estratégias de abordagem familiar e social. Ainda, abaixo do esperado, encontraram-se ações de prescrição de medicamentos em acordo com os protocolos de cuidado, educação para qualificação do cuidado, agendamento e coordenação de encaminhamentos.

Na tabela 3, apresentam-se os dados referentes aos cuidados específicos do enfermeiro na APS, convergentes com o processo de trabalho.

Tabela 3. Cuidados específicos realizados pelos enfermeiros da Atenção Primária à Saúde. Cascavel, Paraná, Brasil. 2017

\begin{tabular}{|c|c|c|c|c|c|c|c|c|c|c|c|c|}
\hline \multirow[b]{3}{*}{ ATIVIDADES } & \multicolumn{6}{|c|}{ Unidade Básica } & \multicolumn{6}{|c|}{ Saúde da Família } \\
\hline & \multicolumn{2}{|c|}{ SIM } & \multicolumn{2}{|c|}{ NÃO } & \multicolumn{2}{|c|}{ Parcial } & \multicolumn{2}{|c|}{ SIM } & \multicolumn{2}{|c|}{ NÃO } & \multicolumn{2}{|c|}{ Parcial } \\
\hline & $\mathbf{N}$ & $\%$ & $\mathbf{N}$ & $\%$ & $\mathrm{~N}$ & $\%$ & $\mathbf{N}$ & $\%$ & $\mathrm{~N}$ & $\%$ & $\mathbf{N}$ & $\%$ \\
\hline $\begin{array}{l}\text { Acompanha crescimento e desenvolvimento } \\
\text { da criança até dois anos }\end{array}$ & 9 & $75 a$ & 0 & 0 & 3 & 25 & 11 & $92 a$ & 0 & 0 & 1 & 8 \\
\hline $\begin{array}{l}\text { Avalia crescimento e desenvolvimento em } \\
\text { recém-nascido prematuro e com baixo peso } \\
\text { ao nascer }\end{array}$ & 8 & $67 b$ & 1 & 8 & 3 & 25 & 9 & $75 a$ & 0 & 0 & 3 & 25 \\
\hline $\begin{array}{l}\text { Monitora encaminhamento de consulta } \\
\text { odontológica no primeiro ano de vida }\end{array}$ & 6 & $50 b$ & 4 & 33 & 2 & 17 & 8 & $67 b$ & 1 & 8 & 3 & 25 \\
\hline $\begin{array}{l}\text { Identifica crianças beneficiadas pelo bolsa } \\
\text { família }\end{array}$ & 5 & $42 c$ & 1 & 8 & 5 & 42 & 9 & $75 b$ & 2 & 17 & 1 & 8 \\
\hline Desenvolve pré-natal & 5 & $42 c$ & 1 & 8 & 6 & 50 & 8 & $67 b$ & 0 & 0 & 4 & 33 \\
\hline Captação da gestante no primeiro trimestre & 7 & $58 b$ & 0 & 0 & 5 & 42 & 6 & $50 b$ & 1 & 8 & 5 & 42 \\
\hline $\begin{array}{l}\text { Atende puérpera e recém-nascido na primei- } \\
\text { ra semana de vida }\end{array}$ & 5 & $42 c$ & 1 & 8 & 6 & 50 & 9 & $75 b$ & 0 & 0 & 3 & 25 \\
\hline Ações de prevenção do câncer do colo uterino & 12 & $100 a$ & 0 & 0 & 0 & 0 & 11 & $92 a$ & 0 & 0 & 1 & 8 \\
\hline Identifica risco/ações de doenças crônicas & 6 & $50 b$ & 4 & 33 & 2 & 17 & 4 & $33 c$ & 1 & 8 & 7 & 58 \\
\hline Vigilância tuberculose e hanseníase & 5 & $42 c$ & 1 & 8 & 6 & 50 & 11 & $92 a$ & 0 & 0 & 1 & 8 \\
\hline Planejamento familiar e saúde sexual & 2 & $17 c$ & 4 & 33 & 6 & 50 & 6 & $50 b$ & 0 & 0 & 6 & 50 \\
\hline $\begin{array}{l}\text { Diagnóstico e acompanhamento de infec- } \\
\text { ções sexualmente transmissíveis e Aids }\end{array}$ & 6 & $50 b$ & 2 & 17 & 4 & 33 & 5 & $42 c$ & 1 & 8 & 6 & 50 \\
\hline Vigilância em saúde ambiental & 7 & $58 b$ & 1 & 8 & 4 & 33 & 9 & $75 b$ & 0 & 0 & 3 & 25 \\
\hline Investigação de óbitos infantis & 11 & $92 a$ & 0 & 0 & 1 & 8 & 10 & $83 a$ & 0 & 0 & 2 & 17 \\
\hline $\begin{array}{l}\text { Disponibiliza informações sobre a unidade } \\
\text { aos usuários }\end{array}$ & 11 & $92 a$ & 0 & 0 & 1 & 8 & 11 & $92 a$ & 0 & 0 & 1 & 8 \\
\hline
\end{tabular}

Fonte: Elaboração própria.

(a) executa as ações em acordo com o processo de trabalho - médias de 71 a 100; (b) executa as ações parcialmente em acordo com o processo de trabalho - médias de 50 a 70; (c) não executa as ações em acordo com o processo de trabalho - médias menores que 49

Entre as atividades que sobressaem nos dois modelos de atenção, na métrica $\mathrm{A}$, tem-se o acompanhamento do crescimento e do desenvolvimento da criança até os dois anos, as ações de prevenção do câncer de colo cervicouterino, a investigação dos óbitos infantis e a disponibilização de informações sobre o funcionamento da unidade aos usuários.

Como atividades desenvolvidas, contudo de forma menos frequente, agrupadas na métrica B, e de forma similar entre os modelos de atenção, obtiveram-se o monitoramento de 
encaminhamentos para a consulta odontológica no primeiro ano de vida, a captação da gestante no primeiro trimestre e a vigilância em saúde ambiental. De forma distinta, com predominância na ESF, encontraram-se avalição do crescimento e desenvolvimento da criança prematura e com baixo peso ao nascer, identificação e acompanhamento de crianças beneficiadas pelo bolsa família, o desenvolvimento de pré-natal, atenção à puérpera e ao recém-nascido na primeira semana de vida, vigilância da tuberculose e hanseníase e ações de planejamento familiar e de saúde sexual. Ao contrário, mesmo que na métrica $\mathrm{B}$, com proeminência da UBS, perceberam-se a identificação de risco dos usuários com condições crônicas e as ações para o diagnóstico e acompanhamento de HIV/Aids e infecções sexualmente transmissíveis.

Na tabela 4, encontram-se as atividades do processo de trabalho elencadas na métrica $\mathrm{C}$, ou seja, pouco desenvolvidas no cotidiano dos enfermeiros na $\mathrm{AB}$.

Tabela 4. Cuidados específicos pouco realizados pelos enfermeiros da Atenção Primária à Saúde. Cascavel, Paraná, Brasil. 2017

\begin{tabular}{|c|c|c|c|c|c|c|c|c|c|c|c|c|}
\hline \multirow[b]{3}{*}{ ATIVIDADES } & \multicolumn{6}{|c|}{ Unidade Básica } & \multicolumn{6}{|c|}{ Saúde da Família } \\
\hline & \multicolumn{2}{|c|}{ SIM } & \multicolumn{2}{|c|}{ NÃO } & \multicolumn{2}{|c|}{ Parcial } & \multicolumn{2}{|c|}{ SIM } & \multicolumn{2}{|c|}{ NÃO } & \multicolumn{2}{|c|}{ Parcial } \\
\hline & $\mathbf{N}$ & $\%$ & $\mathbf{N}$ & $\%$ & $\mathbf{N}$ & $\%$ & $\mathbf{N}$ & $\%$ & $\mathrm{~N}$ & $\%$ & $\mathbf{N}$ & $\%$ \\
\hline Acompanha crianças com até 9 anos & 0 & Oc & 8 & 67 & 4 & 33 & 1 & $8 c$ & 6 & 50 & 5 & 42 \\
\hline Acompanha as gestantes do território & 1 & $8 c$ & 4 & 33 & 7 & 58 & 2 & $17 \mathrm{c}$ & 1 & 8 & 9 & 75 \\
\hline $\begin{array}{l}\text { Solicita exames para as gestantes e realiza } \\
\text { consulta de pré-natal }\end{array}$ & 5 & $42 c$ & 2 & 17 & 5 & 42 & 4 & $33 c$ & 2 & 17 & 6 & 50 \\
\hline Educação em saúde as gestantes & 1 & $8 c$ & 7 & 58 & 4 & 33 & 2 & $17 c$ & 0 & 0 & 10 & 83 \\
\hline Ações com portadores de doenças crônicas & 2 & $17 \mathrm{c}$ & 5 & 42 & 5 & 42 & 5 & $42 c$ & 2 & 17 & 5 & 42 \\
\hline $\begin{array}{l}\text { Identifica e acompanha casos da saúde } \\
\text { mental }\end{array}$ & 0 & Oc & 6 & 50 & 6 & 50 & 4 & $33 c$ & 2 & 17 & 6 & 50 \\
\hline Identifica casos de violência & 1 & $8 c$ & 6 & 50 & 5 & 42 & 4 & $33 c$ & 1 & 8 & 7 & 58 \\
\hline Ações de saúde com os trabalhadores & 3 & $25 c$ & 3 & 25 & 6 & 50 & 5 & $42 c$ & 4 & 33 & 3 & 25 \\
\hline $\begin{array}{l}\text { Ações de saúde física, alimentaccão saudável, } \\
\text { fitoterápicos }\end{array}$ & 0 & Oc & 11 & 92 & 1 & 8 & 0 & Oc & 7 & 58 & 5 & 42 \\
\hline
\end{tabular}

Fonte: Elaboração própria.

(a) executa as ações em acordo com o processo de trabalho - médias de 71 a 100; (b) executa as ações parcialmente em acordo com o processo de trabalho - médias de 50 a 70; (c) não executa as ações em acordo com o processo de trabalho - médias menores que 49.

As atividades elencadas fazem parte de programas de saúde, mas não receberam prioridade pelos enfermeiros do estudo, sendo classificadas na métrica C, embora integrem programas de saúde relativos a APS, como o seguimento da criança e da gestante, a atenção a condição crônica, cuidados para a saúde mental, prevenção de violências, saúde do trabalhador e práticas mais abrangentes e integrativas para promoção da saúde.

\section{Discussão}

A enfermagem no Brasil, como campo de conhecimento, é composta pelas categorias Enfermeiros, Técnicos de Enfermagem e Auxiliares de Enfermagem 7 , cujos processos de trabalho são também distintos, ainda que interdependentes. Essa configuração, denominada Enfermagem, dificulta a identificação do trabalho do enfermeiro no interior da profissão 
e também no conjunto das profissões da área da saúde, uma vez que esse profissional realiza um trabalho no campo da APS que se assemelha ao trabalho em saúde de uma forma genérica, em que são socializadas, com outros profissionais, práticas de saúde consideradas comuns e pertinentes a qualquer categoria profissional, na qual são priorizadas ações do campo da área coletiva em detrimento das ações nucleares da profissão, havendo um distanciamento de seu fazer específico, que poderia ser um referencial identitário ${ }^{8}$.

No artigo em tela, a APS, entendida como o primeiro nível de atenção, é caracterizada por um conjunto de ações nos âmbitos individual e coletivo, que abrangem a promoção e a proteção da saúde, a prevenção de agravos, o diagnóstico, o tratamento, a reabilitação e a manutenção da saúde ${ }^{6}$, confirmando as práticas de saúde coletiva descritas.

Diante desse escopo, o enfermeiro da APS, para executar essa diversidade de ações comuns e específicas que lhe são atribuídas ${ }^{2}$, necessita desenvolver várias competências, entre elas, a habilidade de desempenhar a consulta de enfermagem para conseguir produzir o cuidado e a gestão do processo terapêutico, bem como atividades de gerenciamento do serviço de saúde e da equipe de enfermagem, as quais se caracterizam como as funções predominantes de seu trabalho na APS. Assim, discutem-se as ações que o enfermeiro desenvolve de acordo com a realidade estudada, tanto como integrante da equipe de saúde quanto as suas atribuições específicas.

\section{Atividades do enfermeiro como integrante da equipe interdisciplinar}

Evidenciou-se na prática predominante do enfermeiro como integrante da equipe de saúde, na UBS, enfoque mais voltado a ações de atenção à saúde para um território definido, a exemplo de estudo que considera o território um espaço geográfico com amplas características, em permanente construção e reconstrução, no qual os diversos atores se deparam com distintas necessidades de saúde, para as quais a oferta de serviços é insuficiente, pois as demandas existentes ultrapassam os limites do setor'.

Consoante, estudo realizado com equipes de ESF verificou o diagnóstico situacional do território como um instrumento importante para a realização de ações prioritárias. Utilizar a territorialização para conhecer as demandas da população possibilita o conhecimento das condições de saúde dos grupos etários e as vulnerabilidades do território ${ }^{10}$. Muito embora a territorialização seja um instrumento de trabalho habitualmente vinculado a ESF, nesta pesquisa, ele esteve presente também no trabalho do enfermeiro da UBS.

Na ESF, neste estudo, evidenciou-se o predomínio de ações como acolhimento, reuniões de equipe, identificação das necessidades de intervenções de cuidado e educação permanente em saúde, todos temas sensíveis à prática do enfermeiro nesse modelo de cuidado. Ao contrário desses achados, em estudo realizado em Campinas (SP), os enfermeiros afirmaram que não reconhecem o acolhimento como parte do cuidado realizado pelo enfermeiro que atua na APS, e que, na prática, o acolhimento é uma ação distante, pois veem tal prática sendo direcionada apenas à demanda espontânea do serviço de saúde"1.

Para avaliar o acolhimento, estudo realizado com 10 UBS de Brasília encontrou que a maioria dos enfermeiros daquelas unidades considera que o trabalho realizado nelas é focado na consulta médica, e que o atendimento à demanda espontânea é realizado por meio da distribuição de fichas. Com isso, verifica-se que o acolhimento não é realizado, pois, ao estabelecer o número de fichas para atendimento médico, acabam-se excluindo os usuários que não tiveram acesso a elas, assim como se perde a oportunidade de realizar a escuta qualificada de suas necessidades, as quais poderiam ser atendidas por outros profissionais da equipe, como o enfermeiro, bem como dificulta a criação de vínculo com a população ${ }^{12}$. 
Ainda acerca do acesso dos usuários à APS, estudo realizado com 323 equipes de ESF e 1.313 usuários das unidades no Rio de Janeiro questionou os usuários sobre qual serviço de saúde procuraram na última vez que tiveram algum problema de saúde, e $73 \%$ deles responderam a UBS, $84,2 \%$ referiram ter sido atendidos na mesma hora na unidade, embora 18,1 tenham conseguido a consulta médica demandada para o mesmo dia. Além disso, $99 \%$ das equipes afirmaram realizar acolhimento e atendimento a demanda espontânea todos os dias da semana, sendo o enfermeiro citado por 98,4\% como o profissional que mais participa e realiza acolhimento na unidade ${ }^{\mathbf{1 3}}$. Ademais, o acolhimento permite identificar as necessidades de saúde da população ${ }^{\mathbf{1 4}}$.

Outro aspecto que sobressaiu na ESF, as reuniões de equipe, foi tratado em estudo ${ }^{15}$ para evidenciar a estrutura, o funcionamento e as contribuições das reuniões de trabalho da ESF para o planejamento local em saúde, tendo encontrado que as reuniões de trabalho são momentos indispensáveis para construção do planejamento local em saúde. Além disso, proporcionam a integração dos profissionais por meio de discussões, facilitando a tomada de decisões e a troca de informações.

Ainda, foi evidenciada a educação permanente em saúde, corroborando pesquisa realizada com profissionais de ESF no município de Uberaba, em que os gestores das unidades a mencionam como ferramenta utilizada tanto para formação da população quanto dos profissionais, pois podem realizar a avaliação de ambos, destacar os problemas enfrentados e transformá-los em conhecimento por meio da capacitação para as equipes propiciarem a educação em saúde ${ }^{16}$.

Em ambos os modelos de atenção, encontraram-se ações de promoção, prevenção, busca ativa, trabalho interdisciplinar, registro de atividades no Siab, os quais são elementos característicos da APS, de forma similar a estudo que menciona as características nucleares da profissão, tais como o acompanhamento periódico da população do seu território adscrito, organização e supervisão dos serviços da unidade e o empoderamento dos atores sociais por meio das ações educativas ${ }^{8}$.

Quanto ao registro de atividades, embora com menor percentual (67\%), foi mais reportado pelos enfermeiros da ESF, indicando que o trabalho é desenvolvido, mas não registrado na mesma medida em que ocorre. Para entender por que o registro não é realizado, estudo demonstrou que os profissionais não têm confiança em registrar as informações em sistema on-line e, muitas vezes, acabam utilizando conjuntamente outras fontes de registros. Os enfermeiros expressaram que a equipe deve ter mais controle do registro realizado e que eles costumam comparar as informações de outras fontes de registro para realizar seus atendimentos ${ }^{17}$.

Sobre as ações do enfermeiro como integrante da equipe de saúde, nesta pesquisa, houve aquelas que são realizadas com pouca frequência, destacando-se o cadastro das famílias e as ações intersetoriais, além do uso de dados epidemiológicos para o planejamento de atividades em saúde pela equipe interdisciplinar. O cadastro das famílias no território possibilita estabelecer o perfil epidemiológico da população adscrita. Assim, como o enfermeiro não tem realizado ou participado dessa atividade, por consequência, não dispõe de dados epidemiológicos para planejar suas atividades.

Estudo menciona que essa ação deve ser executada por todos os membros da equipe, para que conheçam e identifiquem os problemas e as necessidades da população e desenvolvam estratégias para o atendimento e a mudança dessas condições, pois, quando a unidade de saúde realiza o planejamento utilizando dados reais da população, a equipe de ESF se destaca na APS, uma vez que possui melhores resultados por utilizar a análise situacional de seu território ${ }^{17}$.

As ações intersetoriais, por sua vez, podem auxiliar a resolver os problemas oriundos da determinação social do processo saúde-doença, e não têm sido desenvolvidas. Sobre 
o tema, estudo da área da saúde do trabalhador identifica a intersetorialidade como uma construção cotidiana, a partir da ênfase nos aspectos comuns que transcendem o corporativismo de cada categoria ou setor. Apontam como dificuldades para a sua construção, entre os diferentes setores, o desconhecimento dos papéis uns dos outros, não havendo visibilidade das ações desenvolvidas e compartilhamento de experiências ${ }^{\mathbf{1 8}}$.

\section{Atribuições específicas do trabalho do enfermeiro na APS}

No que tange às atividades específicas do trabalho do enfermeiro na APS, depreende-se a atuação gerencial predominando sobre a prática clínica, priorizando-se atividades como coordenação dos ACS, em ambos os modelos de atenção, práticas de educação permanente em saúde e participação no gerenciamento de insumos. Por outro lado, ações de prática clínica, como a assistência permeada por protocolos, os quais embasam a solicitação de exames e terapêutica, são desempenhadas parcialmente, com a solicitação de exames sem o devido acompanhamento do usuário pelo profissional, inclusive sem o uso de ferramentas de gestão do cuidado para casos complexos.

Ademais, os enfermeiros de ambos os modelos de atenção, mas ressaltando-se o enfermeiro de USF, para os quais essas práticas eram esperadas, não utilizam as práticas educativas e ferramentas de tecnologia do cuidado em saúde para atenção aos usuários da APS, além da ausência de diagnóstico situacional para o planejamento das ações de cuidado, da pouca participação na elaboração da agenda de cuidados da unidade, do não acompanhamento dos usuários na rede de cuidados após os encaminhamentos e, principalmente, da não utilização de instrumentos de abordagem familiar e comunitária na APS.

A despeito do elencado, para o cuidado como seu objeto de trabalho, o enfermeiro necessita do diálogo, respeito e humanização. O cuidado em saúde pode ser praticado em qualquer ambiente, desde que o enfermeiro consiga desenvolver um plano de cuidado, a partir do conhecimento do indivíduo, de sua família, habitação, condição de saúde ${ }^{\mathbf{1 4}}$, o que precisa ser mais bem aplicado na realidade do estudo.

Um dos espaços utilizados pelo enfermeiro para a atenção à saúde na APS aos indivíduos e à família é o domicílio, opção que se mostrou realidade para $83 \%$ dos enfermeiros nesta pesquisa, em ambas as unidades. Essa prática vai além da realização de atividades assistenciais, pois se utiliza desse momento de contato entre enfermeiro e indivíduo para realizar procedimentos e, ao mesmo tempo, para examinar o paciente, realizar escuta ativa, aplicar ações de educação em saúde e criar um vínculo entre unidade e comunidade ${ }^{19}$.

Com relação à consulta de enfermagem, estudo de revisão sobre o tema ${ }^{20}$ indica que foi com a implementação da ESF que essa prática se tornou popular para os usuários e demais profissionais da APS, evidenciando que a consulta de enfermagem é bem vista pelos usuários devido ao seu caráter educativo, proporcionando autonomia ao usuário, melhorando sua qualidade de vida e o vínculo dos usuários com o enfermeiro, a equipe e a unidade de saúde. Ademais, a partir da implementação das consultas de enfermagem, ocorreu a ampliação do acesso aos usuários ${ }^{21}$.

Em estudo 22 realizado a partir do Programa de Melhoria do Acesso e da Qualidade (PMAQ), abrangendo 3.972 municípios de todos os estados da Federação, no qual os enfermeiros foram a maioria dos entrevistados, ao se avaliar a realização de agendamento de consultas, a maior quantidade de profissionais que o executam está em Minas Gerais (MG), ainda que a porcentagem seja baixa, pois, nos demais estados, poucos enfermeiros participam do agendamento, como evidenciado nos dados desta pesquisa.

Existem dois pontos abordados em outros estudos que sugerem a explicação para que os enfermeiros não consigam monitorar e encaminhar os usuários. Em pesquisa realizada 
com profissionais de saúde pública, enfermeiros informaram que muitas vezes perdem os usuários quando estes são encaminhados a outros setores devido à falta de comunicação e de informação das outras redes ${ }^{23}$. Em outro estudo, realizado em Centros de Saúde de Santa Catarina, os profissionais relataram que, pela falta de tempo e falta de comunicação, existem muitos encaminhamentos que acontecem apenas pelos funcionários da recepção, em que não há avaliação clínica dos usuários, e, muitas vezes, esse atendimento não resolve os seus problemas ${ }^{\mathbf{2 4}}$.

Verificou-se, ainda, que a utilização de ferramentas na gestão do cuidado também foi pouco referenciada pelos enfermeiros de nossa pesquisa (33\%). Quando se utiliza o termo ferramentas ou tecnologias para a gestão do cuidado, refere-se, também, a processos de trabalho que se somam a essa prática, como, por exemplo, a utilização do acolhimento, construção de vínculo com o usuário e a realização de diagnósticos e prescrições de enfermagem ${ }^{25}$.

Outro aspecto pouco desempenhado no cuidado foi a prescrição de medicamentos guiada por protocolos de cuidado, evidenciando a pouca autonomia do enfermeiro para o cuidado clínico. A esse respeito, estudo realizado com sete enfermeiras, em seis unidades de APS, em Londres, Inglaterra, demonstra que a atuação dos profissionais refere-se a práticas clínicas, manejo de casos crônicos e prescrição de medicamentos, em que o atendimento das condições crônicas é pautado em protocolos de cuidado que ampliam a autonomia e o processo de trabalho dos enfermeiros ${ }^{26}$.

\section{Cuidados específicos realizados pelos enfermeiros aos usuários da APS}

Embora o cuidado em saúdem na APS seja compartilhado, exercer o cuidado é uma das competências do enfermeiro, que vai além do conhecimento técnico e deve integrar-se às práticas desenvolvidas por todos os profissionais do serviço de saúde. O enfermeiro é um dos profissionais responsáveis por prestar o cuidado integral nas ações da APS, para que o envolvimento com os usuários não seja apenas focado na doença e no tratamento, mas considere as condições que permeiam a vida do indivíduo ${ }^{19}$.

A respeito desse fazer do enfermeiro na APS, estudo menciona que esse profissional possui uma visão integrativa e ampliada sobre as ações na ESF, mas não consegue implementá-las devido à lógica de produtividade no atendimento instituído nas unidades, pela grande concentração de consultas de enfermagem, sobretudo o pré-natal ${ }^{27}$.

Como já visto neste estudo, as ações mais desempenhadas pelos enfermeiros em ambos os modelos incluem a atenção à criança na puericultura, até os dois anos, prevenção do câncer de colo de útero, investigação de óbitos infantis e disponibilização de informações aos usuários da unidade. Com menos frequência, mas ainda assim executados, estão o encaminhamento para avaliação odontológica de rotina, a captação das gestantes no primeiro trimestre e ações de saúde ambiental.

Houve predomínio de ações sendo desenvolvidas pelo enfermeiro da ESF, como seguimento de prematuro, acompanhamento das crianças do programa Bolsa Família, desenvolvimento de pré-natal, avaliação dos pacientes em programas de saúde, como de tuberculose e hanseníase, e planejamento familiar. $\mathrm{Na}$ UBS, as ações do enfermeiro que sobressaíram foram a identificação de risco para condição crônica e o acompanhamento de pacientes nas doenças sexualmente transmissíveis.

Convergindo com esses dados, estudo ${ }^{8}$ aborda os elementos que refletem a essência da profissão do enfermeiro, como a prática da clínica, semiologia e semiotécnica associadas com o acolhimento, vínculo, escuta, expressas, por exemplo, por meio da consulta de enfermagem e ações educativas, as quais podem possibilitar mais autonomia, bem como capital simbólico, ou seja, mais reconhecimento, credibilidade e visibilidade ao enfermeiro no campo da APS, que muito faz, mas que nem sempre tem suas ações evidenciadas no campo da saúde. 
Some-se a isso que os enfermeiros na APS vêm conquistando espaço social e reconhecimento junto aos integrantes da equipe de saúde e dos usuários, que vivenciam com eles o atendimento clínico e identificam neles a referência para o seu cuidado, o que traz satisfação e dá sentido ao trabalho. Os enfermeiros reconhecem, em seu fazer, potencialidades, como: exercer a prática clínica por meio da consulta de enfermagem, criar vínculos com a população e estabelecer com a equipe relações interpessoais que propiciem um ambiente de trabalho produtivo, saudável e satisfatório, destacando aspectos importantes para a realização de suas atribuições, como a valorização e o reconhecimento, educação permanente em saúde, vínculo estabelecido com a comunidade e trabalho conjunto 8 .

Para além dessas atividades, estudo realizado com 32 enfermeiros de USF no município de Caxias (MA) aponta que as ações realizadas nas unidades têm cunho gerencial, sendo que a organização de programas sociais e a disponibilidade de informações foram citadas como práticas de articulação entre os serviços do município. Abordam que o desenvolvimento das ações depende muito da supervisão dos enfermeiros, da autonomia desse profissional dentro da unidade, do provimento de recursos para que a equipe possa desenvolver os programas na unidade 28 .

Ao contrário, determinadas ações foram pouco ou não desenvolvidas pelos enfermeiros, como o seguimento das crianças após os dois anos, o acompanhamento das gestantes, com solicitação de exames e realização do pré-natal e educação em saúde para as gestantes, as ações de cuidado a pessoas com condições crônicas, atenção à saúde mental e violências, saúde do trabalhador e práticas integrativas e complementares. Segundo a $\mathrm{PNAB}^{2}$, o enfermeiro tem como atribuição desenvolver a estratificação de risco das pessoas de seu território e desenvolver um plano de cuidado àqueles que possuem casos crônicos, conjuntamente com os outros profissionais da equipe.

Para além de identificar, é necessário realizar ações de cuidado ao público portador de doenças crônicas, contudo, apenas $17 \%$ dos enfermeiros de UBS deste estudo a realizam, com uma porcentagem um pouco maior na USF (42\%). Ao contrário, em estudo realizado no Rio de Janeiro (RJ), os enfermeiros realizam ações focadas em grupos, conforme idade, controle de agravos epidemiológicos e doenças crônicas, como hipertensão e diabetes. Utilizam ações educativas como forma de articular as práticas assistenciais de prevenção e de promoção de saúde ${ }^{29}$.

Para o cuidado individual, o enfermeiro pode utilizar instrumentos em suas consultas que facilitem o cuidado integral, como, por exemplo, a utilização da sistematização da assistência através de relatórios, registros, diagnósticos e prescrições de enfermagem aplicadas sob forma de orientações aos usuá$\operatorname{rios}^{25}$, atividade que precisa ser ampliada na realidade da pesquisa, em que predominam as atividades administrativo-burocráticas.

Em convergência com esses dados, pesquisas ${ }^{30,31}$ demonstram que os enfermeiros possuem dificuldades para realizar o atendimento a situações específicas (doenças infecciosas, pessoas em situação de violência, problemas de saúde mental), a exemplo do que foi encontrado neste estudo, mencionando a falta de instrumentos sistematizados, a dificuldade de abordá-los e identificá-los e, ainda, de se responsabilizarem por encaminhar para o cuidado demandado.

Como contribuições para a prática da enfermagem, destacam-se aspectos positivos com relação ao trabalho do enfermeiro na APS, similares para ambos os modelos de atenção, como o acompanhamento do crescimento e desenvolvimento da criança na puericultura, inclusive prematura, as investigações de óbitos infantis e a prevenção do câncer cervicouterino.

Salienta-se que muitas das ações de sua responsabilidade são mais desenvolvidas na ESF do que na UBS, a exemplo do monitoramento e do encaminhamento para as consultas odontológicas do bebê, o seguimento das 
crianças que recebem bolsa família, a captação da gestante no primeiro trimestre da gestação, o atendimento à puérpera e ao $\mathrm{RN}$ na primeira semana de vida, fazendo emergir a preocupação de que essas ações possam se perder a partir da nova PNAB. Ademais, embora com menor frequência, também se realizam identificação e estratificação de condições crônicas, planejamento familiar e saúde sexual, diagnóstico e acompanhamento de casos de Infecções Sexualmente Transmissíveis (IST) e Síndrome da Imunodeficiência Adquirida (Aids) e vigilância ambiental.

Por outro lado, ações importantes tanto na ESF quanto na UBS ou não são desenvolvidas ou muito poucos enfermeiros responderam que as adotam, tais como o seguimento da criança até os nove anos, o acompanhamento das gestantes de sua área adscrita, a solicitação de exames para as gestantes ou a realização do acompanhamento do pré-natal de risco habitual, educação em saúde para gestantes, identificação de casos de violência e ações de saúde do trabalhador, fazendo emergir a preocupação com a realização de novos estudos sobre o processo de trabalho do enfermeiro na APS.

Como limitação, aponta-se que o estudo abordou somente a descrição das atividades no trabalho do enfermeiro, em correspondência ao que lhe é atribuído pela PNAB. Outros estudos que investiguem as condições de trabalho do enfermeiro, a estrutura das unidades, a organização do trabalho, são necessários para compreender por que determinadas ações sobressaem no conjunto das atividades.

\section{Conclusões}

Requer-se do enfermeiro na atenção primária que desempenhe um conjunto de atividades tanto integrando a equipe de saúde quanto as do seu próprio fazer profissional. $\mathrm{O}$ estudo evidenciou que o conjunto de demandas exigidas ao enfermeiro não é plenamente executado. A PNAB, recentemente modificada, aponta como lócus do cuidado na APS os dois tipos de unidades, os quais, como demonstrado neste estudo, necessitam de reformulação na forma como organizam seus serviços, face a evidente dificuldade dos enfermeiros em desempenhar seu processo de trabalho conforme disposto na resolução que orienta a prática profissional na APS.

Compreende-se que, diante das inúmeras atribuições do enfermeiro, como integrante da equipe de saúde e as suas funções específicas, parece estar havendo predomínio em atividades que poderiam ser desempenhadas por outros membros da equipe, enquanto as atividades próprias da práxis do enfermeiro estão em segundo plano. Sugere-se uma reflexão sobre o processo de trabalho com vistas à apropriação de sua área de saber e desempenho de funções específicas, evidenciando o protagonismo do enfermeiro, que hoje pouco sobressai nesse âmbito de atenção.

\section{Colaboradoras}

Toso BRGO (0000-0001-7366-077X)* contribui para o desenho do estudo, redação do relatório de pesquisa, redação do artigo e aprovação de sua versão final. Fungueto L (0000-0001-8888-5656)* contribui para a elaboração do projeto de pesquisa, coleta de dados, organização e análise dos dados, elaboração do manuscrito e revisão de sua versão final. Maraschin MS (0000-0003-21845056)* e Tonini NS (0000-0003-4704-7634)* contribuíram igualmente para a elaboração do manuscrito. 


\section{Referências}

1. Starfield B, Shi L, Macinko J. Contribution of primary care to health systems and health. The Milbank Quarterly. 2005; 83(3):457-502.

2. Brasil. Ministério da Saúde. Portaria no 2.436 estabelece a revisão de diretrizes da Política Nacional de Atenção Básica (PNAB). 2017 [acesso em 2017 out 6]. Disponível em: http://www.foa.unesp.br/home/pos/ ppgops/portaria-n-2436.pdf.

3. Paim JS. Modelos de atenção à saúde no Brasil. In: Giovanella L, organizadora. Políticas e sistema de saúde no Brasil. Rio de Janeiro: Ed. Fiocruz; 2012.

4. Morosini MVGC, Fonseca AF, Lima LD. Política Nacional de Atenção Básica 2017: retrocessos e riscos para o Sistema Único de Saúde. Saúde debate. 2018; 42(116):11-24.

5. Brandão LGVA, Teixeira CC, Afonso TC, et al. O sentido do trabalho na Atenção Primária à Saúde. REAS. 2019; 11(8):e528.

6. Ferreira SRS, Perico LAD, Dias VRGF. The complexity of the work of nurses in Primary Health Care. Rev. Bras. Enferm. 2018; 71(sup1):704-9.

7. Brasil. Lei $\mathrm{n}^{\circ}$ 7.498, de 25 de junho de 1986: dispõe sobre a regulamentação do exercício da enfermagem e dá outras providências. Diário Oficial da República Federativa do Brasil, Brasília, DF, Seção 1. p. 1-4; 1986.

8. Fernandes MC, Silva LMS, Silva MRF, et al. Identity of primary health care nurses: perception of "doing everything”. Rev. Bras. Enferm. 2018; 71(1):142-7.

9. Pinto AGA, Jorge MSB, Marinho MNASB, et al. Experiences in the Family Health Strategy: demands and vulnerabilities in the territory. Rev. Bras. Enferm. 2017; 70(5):920-7.

10. Pinto AGA, Sampaio KR, Lobo AC, et al. Grupos prioritários da estratégia saúde da família: a atenção primária à saúde na prática. J. nurs. health. 2016 [acesso em 2020 abr 17]; 6(3):366-378. Disponível em: http:// docs.bvsalud.org/biblioref/bdenf/2016/bde-31740/ bde-31740-595.pdf.

11. Costa PCP, Garcia APRF, Toledo VP. Welcoming and nursing care: a phenomenological study. Texto \& contexto enferm. 2016; 25(1):e4550015.

12. Camelo MS, Lima LR, Volpe CRG, et al. Welcoming in primary health care from the viewpoint of nurses. Acta Paul. Enferm. 2016; 29(4):463-468.

13. Almeida PF, Marin J, Casotti E. Estratégias para consolidação da coordenação do cuidado pela atenção básica. Trab. educ. saúde. 2017; 15(2):373-398.

14. Santos DS, Mishima SM, Merhy EE. Processo de trabalho na Estratégia de Saúde da Família: potencialidades da subjetividade do cuidado para reconfiguração do modelo de atenção. Ciênc. Saúde Colet. 2018; 23(3):861-870.

15. Voltolini BC, Andrade SR, Piccoli T, et al. Estratégia saúde da família meetings: an indispensable tool for local planning. Texto \& contexto enferm. 2019; (28):e20170477.

16. Martins RAS, Souza CAA. Health education in the context of primary health care. REFACS. 2017; 5(2):282288.

17. Senna MH, Andrade SR. Indicators and information in local health planning: the perspective of the family health strategy nurses. Texto \& contexto enferm. 2015; 24(4):950-958.

18. Barros JO, Daldon MTB, Rocha TO, et al. Intersectoriality in health and work in the current brazilian context: the utopia of reality? Interface (Botucatu). 2020; 24:e190303.

19. Acioli S, Kebiani LVA, Faria MGA, et al. Care practices: the role of nurses in primary health care. Rev. enferm. UERJ. 2014; 22(5):637-642. 
20. Maranha NB, Silva MCA, Brito IC. A consulta de enfermagem no cenário da atenção básica e a percepção dos usuários: revisão integrativa. Academus Rev Cient. da Saúde. 2017 [acesso em 2020 maio 2]; 2(1):110. Dis ponível em: https://smsrio.org/revista/index. php/reva/article/view/246/261.

21. Lowen IMV, Peres AM, Ros C, et al. Innovation in nursing health care practice: expansion of access in primary health care. Rev. Bras. Enferm. 2017; 70(5):898903.

22. Galavote HS, Zandonade E, Garcia ACP, et al. The nurse's work in primary health care. Esc. Anna Nery Rev. Enferm. 2016; 20(1):90-98

23. Maximino VS, Liberman F, Frutuoso MF, et al. Profissionais como produtores de redes: tramas e conexões no cuidado em saúde. Saúde Soc. 2017; 26(2):435-447.

24. Souza TH, Zeferino MT, Fermo VC. Reception: strategic point for user access to the unified health system. Texto \& contexto enferm. 2016; 25(3):e4440015.

25. Mororó DDS, Enders BC, Lira ALBC, et al. Concept analysis of nursing care management in the hospital context. Acta Paul. Enferm. 2017; 30(3):323-332.

26. Toso BRGO, Filippon J, Giovanella L. Nurses' performance on primary care in the National Health Service in England. Rev. Bras. Enferm. 2016; 69(1):169-77.
27. Ramos CFV, Araruna RC, Lima CMF, et al. Education practices: research-action with nurses of Family Health Strategy. Rev. Bras. Enferm. 2018; 71(3):1144-51.

28. Silva RNA, Lima AKM, Filha FSSC, et al. Conhecimento e entendimento de enfermeiros sobre as ações gerenciais na atenção primária à saúde. Ciênc. saúde. 2016; 9(1):21-29.

29. Tavares RE, Tocantins FR. Nursing actions in primary care and the control of diseases preventable through vaccines. Rev. Bras. Enferm. 2015; 68(5):803-809.

30. Zambenedetti G, Silva RAN. Descentralização da atenção em HIV-Aids para a atenção básica: tensões e potencialidades. Physis (Rio J.). 2016; 26(3):785806.

31. Egry EY, Apostolico MR, Morais TCP, et al. Coping with child violencein primary care: how do professionals perceive it?. Rev. Bras. Enferm. 2017; 70(1):119125.

\footnotetext{
Recebido em 27/10/2020

Aprovado em 31/05/2021

Conflito de interesses: inexistente

Suporte financeiro: não houve
} 